\title{
Enumerations for Compositions and Complete Homogeneous Symmetric Polynomial
}

\author{
Soumendra Bera ${ }^{1}$ \\ ${ }^{1}$ Production (DHDS), IOCL, P.O.- Haldia Oil Refinery, W.B.721606, India \\ Correspondence: Soumendra Bera, Production (DHDS), IOCL, P.O.- Haldia Oil Refinery, W.B.721606, India. \\ E-mail: soumendra.bera@gmail.com
}

Received: January 12, 2015 Accepted: March 7, 2015 Online Published: March 22, 2015

doi:10.5539/jmr.v7n2p1

URL: http://dx.doi.org/10.5539/jmr.v7n2p1

\begin{abstract}
We count the number of occurrences of $t$ as the summands (i) in the compositions of a positive integer $n$ into $r$ parts; and (ii) in all compositions of $n$; and subsequently obtain other results involving compositions. The initial counting further helps to solve the enumeration problems for complete homogeneous symmetric polynomial.
\end{abstract}

Keywords: composition, summand, sequence, recurrence, binomial coefficient identity, complete homogeneous symmetric polynomial

MS classifications: 05A10; 05A17; 05A19; 11B37

\section{Introduction}

For each nonnegative integer $r$, complete homogeneous symmetric polynomial $h_{r}\left(x_{1}, \ldots, x_{k}\right)$ is the sum of all distinct monomials of degree $r$ in the variables: $x_{1}, \ldots, x_{k}$ Formally

$$
h_{r}\left(x_{1}, \ldots, x_{k}\right)=\sum_{1 \leq i_{1} \leq \ldots \leq i_{r} \leq k} x_{i_{1}} x_{i_{2}} \ldots x_{i_{r}}
$$

Example:

$$
\begin{gathered}
h_{4}\left(x_{1}, x_{2}, x_{3}\right)=x_{1}^{4}+x_{2}^{4}+x_{3}^{4}+x_{1}^{3} x_{2}+x_{1} x_{2}^{3}+x_{1}^{3} x_{3}+x_{1} x_{3}^{3}+x_{2}^{3} x_{3}+x_{2} x_{3}^{3} \\
+x_{1}^{2} x_{2}^{2}+x_{1}^{2} x_{3}^{2}+x_{2}^{2} x_{3}^{2}+x_{1}^{2} x_{2} x_{3}+x_{1} x_{2}^{2} x_{3}+x_{1} x_{2} x_{3}^{2}
\end{gathered}
$$

The number of terms of the polynomial is 15 ; the numbers of occurrences of $1,2,3$ and 4 as the exponents in different terms among 15 terms are 12, 9, 6 and 3 respectively; and the number of occurrences of each of $x_{1}, x_{2}$ and $x_{3}$ as the bases in different terms among 15 terms is 10 .

Evidently $h_{r}\left(x_{1}, \ldots, x_{k}\right)$ has some enumerating problems. We give the solutions of the problems from some combinatorial enumerations for the compositions of a positive integer. The paper has two main parts: (a) counting for compositions and (b) counting for $h_{r}\left(x_{1}, \ldots, x_{k}\right)$.

The main results are as shown:

1. The number of occurrences of an integer $t$ as the summands in the compositions of $n$ into $r$ parts

$$
=r\left(\begin{array}{c}
n-t-1 \\
r-2
\end{array}\right), n \geq r \geq 2, n-r+1 \geq t \geq 1
$$

2. The number of occurrences of $t$ as the summands in all compositions of $n$

$$
=(n-t+3) 2^{n-t-2}, \quad n>t \geq 1
$$

3. The number of occurrences of $t$ as the exponents in different terms among all the terms of $h_{r}\left(x_{1}, \ldots, x_{k}\right)$

$$
=\sum_{i=2}^{r} i\left(\begin{array}{l}
k \\
i
\end{array}\right)\left(\begin{array}{c}
r-t-1 \\
i-2
\end{array}\right), \quad r, k \geq 2, \quad r-1 \geq t \geq 1
$$


4. The number of occurrences of each variable as the bases in different terms among all the terms of $h_{r}\left(x_{1}, \ldots, x_{k}\right)$

\section{Counting for Compositions}

$$
=1+\frac{1}{k} \sum_{t=1}^{r-1} \sum_{i=2}^{r} i\left(\begin{array}{l}
k \\
i
\end{array}\right)\left(\begin{array}{c}
r-t-1 \\
i-2
\end{array}\right)
$$

For counting, we use some simple notations as shown.

1. Compositions of $n$ into $r$ summands $=C(n, r)$.

The notation $C(n, r)$ without any qualification means all compositions of $n$ into $r$ summands. Otherwise we use an adjective to specify the compositions. For example, we write simply 'some $C(n, r)$ ' to mean some compositions of $n$ into $r$ summands.

2. Number of $C(n, r)=N C(n, r)$.

3. Number of occurrences of $t$ in $C(n, r)=N(t) C(n, r)$.

4. Some particular $C(n, r)$ that start with a common summand $k=k+C(n-k, r-1)$.

We use the symbol of equivalence $(\equiv)$ between $C(n, r)$ and its implication; and similarly between $k+C(n-k, r-$ 1) and its implication.

Examples: $C(4,3) \equiv 1+1+2, \quad 1+2+1, \quad 2+1+1$.

$N C(4,3)=3 ; \quad N(1) C(4,3)=6 ; \quad$ and $N(2) C(4,3)=3$.

Some particular $C(6,4)$, which start with a common summand 2 , are:

$2+C(4,3) \equiv 2+1+1+2, \quad 2+1+2+1, \quad 2+2+1+1$.

2.1 Number of Occurrences of $t$ in the Compositions of $n$ into r Parts

The number of the compositions of a positive integer $n$ into $r$ parts or summands is $\left(\begin{array}{c}n-1 \\ r-1\end{array}\right)$. This is a known result. Here we obtain the result in a process of recursive substitution starting with a basic sequential arrangement of $n$ into $r$ summands. The procedure and result lead to count the number of occurrences of $t$ in the compositions of $n$ into $r$ parts.

First we count $N C(n, r)$ for $n \geq r \geq 1$.

(a) By convention, $n$ itself is a composition of $n$ so that $r$ is equal to 1 for the composition. Therefore, for $n \geq 1$ and $r=1$, we have: $N C(n, r)=N C(n, 1)=1$.

(b) For $n \geq r \geq 2$, we can write a basic sequential arrangement of $C(n, r)$ in the following way.

$$
C(n, r) \equiv 1+C(n-1, r-1), \quad 2+C(n-2, r-1), \ldots, \quad(n-r+1)+C(r-1, r-1)
$$

Consequently for $n \geq r \geq 2$,

$$
N C(n, r)=N C(n-1, r-1)+N C(n-2, r-1)+\ldots+N C(r-1, r-1)
$$

(1.1) and (1.2) yield the successive results as shown.

(i) $C(n, 2) \equiv 1+(n-1), 2+(n-2), \ldots, \quad(n-1)+1$.

Hence $N C(n, 2)=n-1$.

(ii) $C(n, 3) \equiv 1+C(n-1,2), \quad 2+C(n-2,2), \ldots, \quad(n-2)+C(2,2)$

Hence $N C(n, 3)=N C(n-1,2)+N C(n-2,2)+\ldots+N C(2,2)$

$=(n-2)+\ldots+1$

$=\left(\begin{array}{c}n-1 \\ 2\end{array}\right)$

Similarly

$N C(n, 4)=N C(n-1,3)+N C(n-2,3)+\ldots+N C(3,3)$

$=\sum_{i=3}^{n-1}\left(\begin{array}{c}i-1 \\ 2\end{array}\right)$

$=\left(\begin{array}{c}n-1 \\ 3\end{array}\right)$ 
In general for $n \geq r \geq 2, N C(n, r)=\left(\begin{array}{c}n-1 \\ r-1\end{array}\right)$; and including the initial result with this, we get:

$$
N C(n, r)=\left(\begin{array}{l}
n-1 \\
r-1
\end{array}\right), n \geq r \geq 1
$$

We count below $N(t) C(n, r)$ by the above process and results.

The basic conditions of $n, t$ and $r$ are: $n \geq t \geq 1, n \geq r \geq 1$ and $r \gtreqless t$.

(a) When $n \geq 1$ and $r=1$ then $t=n$ so that $N(t) C(n, r)=N(n) C(n, 1)=1$. For $n>t \geq 1$, mathematically we can write: $N(t) C(n, 1)=0$. These are the initial results in the counting of $N(t) C(n, r)$.

(b) When $n$ and $r$ are the fixed integers for $n \geq r \geq 2$ then we find some fixed $C(n, r)$. In one of these $C(n, r)$, each of $r-1$ summands is smallest or 1 so that the rest is greatest. Consequently the greatest value of a summand $t$ is $n-r+1$. That is, when $n \geq r \geq 2$ then the condition of $t$ is: $n-r+1 \geq t \geq 1$. From (1.1) we find:

(i) Some $C(n, r)$ start with a common summand $t$; and these are: $t+C(n-t, r-1)$.

(ii) $t$ can occur at other places of different compositions under some or all of $C(n-1, r-1), \quad C(n-2, r-1), \ldots$, $C(r-1, r-1)$.

The smallest positive integer: 1 can occur as the summands in different compositions under $C(n-1, r-1), C(n-$ $2, r-1), \ldots, C(r-1, r-1)$. Yet occurrences of $t \geq 2$ have some limitations. Since $n-r+1 \geq t$, it follows that if $n-r \leq t-2$ then $t$ cannot occur in a $C(n, r)$. More precisely, we cannot find the occurrences of

$$
\begin{aligned}
& 2 \text { in } C(r-1, r-1) \text {; } \\
& 3 \text { in } C(r, r-1) \text { and } C(r-1, r-1) \text {; } \\
& 4 \text { in } C(r+1, r-1), C(r, r-1) \text { and } C(r-1, r-1) \text {; }
\end{aligned}
$$

In general $t$ cannot occur in any composition under $C(r+t-3, r-1), C(r+t-4, r-1), \quad \ldots, C(r-1, r-1)$ for $r \geq 2$ and $t \geq 2$. Other compositions under $C(n-1, r-1), C(n-2, r-1), \ldots, C(r+t-2, r-1)$ may contain $t$. Then from (i) and (ii), we get: for $n \geq r \geq 2$ and $n-r+1 \geq t \geq 1$,

$$
\begin{aligned}
& N(t) C(n, r)=N C(n-t, r-1)+[N(t) C(n-1, r-1)+N(t) C(n-2, r-1)+\ldots \\
&+N(t) C(r+t-2, r-1)] \\
& \Rightarrow N(t) C(n, r)=\left(\begin{array}{c}
n-t-1 \\
r-2
\end{array}\right)+[N(t) C(n-1, r-1)+N(t) C(n-2, r-1)+\ldots \\
&+N(t) C(r+t-2, r-1)]
\end{aligned}
$$

When $r=2$, then for $n \geq 2$ and $n-1 \geq t \geq 1$,

$$
\begin{gathered}
N(t) C(n, 2)=1+N(t) C(t, 1)=1+1 \\
=2
\end{gathered}
$$

Evidently $N(t) C(n, 2)$ is constant and independent of $t$ and $n$. (3.1) is the primary case of (3); and then the rest of (3) is: for $n \geq r \geq 3$ and $n-r+1 \geq t \geq 1$,

$$
\begin{gathered}
N(t) C(n, r)=\left(\begin{array}{c}
n-t-1 \\
r-2
\end{array}\right)+[N(t) C(n-1, r-1)+N(t) C(n-2, r-1)+\ldots \\
+N(t) C(r+t-2, r-1)]
\end{gathered}
$$

Now our aim is to count $N(t) C(n, r)$ applying (3.1) and (3.2).

\section{To count $N(t) C(n, r)$ for $t=1$}

For $n \geq 2, \quad N(1) C(n, 2)=2$

For $n \geq 3, \quad N(1) C(n, 3)=n-2+[N(1) C(n-1,2)+N(1) C(n-2,2)+\ldots+N(1) C(2,2)]$

$$
=3(n-2) \text {. }
$$

For $n \geq 4, \quad N(1) C(n, 4)=\left(\begin{array}{c}n-2 \\ 2\end{array}\right)+[N(1) C(n-1,3)+N(1) C(n-2,3)+\ldots+N(1) C(3,3)]$

$$
\begin{gathered}
=\left(\begin{array}{c}
n-2 \\
2
\end{array}\right)+3[(n-3)+(n-4)+\ldots+1] \\
=4\left(\begin{array}{c}
n-2 \\
2
\end{array}\right) .
\end{gathered}
$$

Similarly for $n \geq 5, N(1) C(n, 5)=\left(\begin{array}{c}n-2 \\ 3\end{array}\right)+4 \sum_{i=4}^{n-1}\left(\begin{array}{c}i-2 \\ 2\end{array}\right)$

$$
=5\left(\begin{array}{c}
n-2 \\
3
\end{array}\right) \text {. }
$$


In general for $n \geq r \geq 2, \quad N(1) C(n, r)=r\left(\begin{array}{c}n-2 \\ r-2\end{array}\right)$

\section{To count $N(t) C(n, r)$ for $t=2$}

$n, r$ and $t$ have the conditions: $n \geq r \geq 2$ and $n-r+1 \geq t \geq 1$. Hence when $t=2$ then the conditions of $n$ and $r$ are: $n-1 \geq r \geq 2$ and $n \geq 3$.

For $n \geq 3, N(2) C(n, 2)=2$.

For $n \geq 4, N(2) C(n, 3)=n-3+[N(2) C(n-1,2)+N(2) C(n-2,2)+\ldots+N(2) C(3,2)]$

$$
=3(n-3) \text {. }
$$

For $n \geq 5, N(2) C(n, 4)=\left(\begin{array}{c}n-3 \\ 2\end{array}\right)+[N(2) C(n-1,3)+N(2) C(n-2,3)+\ldots+N(2) C(4,3)]$

$$
\begin{gathered}
=\left(\begin{array}{c}
n-3 \\
2
\end{array}\right)+3[(n-4)+(n-5)+\ldots+1] \\
=4\left(\begin{array}{c}
n-3 \\
2
\end{array}\right) .
\end{gathered}
$$

Thus for $n \geq 6, N(2) C(n, 5)=\left(\begin{array}{c}n-3 \\ 3\end{array}\right)+4 \sum_{i=5}^{n-1}\left(\begin{array}{c}i-3 \\ 2\end{array}\right)$

In general for $n-1 \geq r \geq 2, N(2) C(n, \quad r)=r\left(\begin{array}{l}n-3 \\ r-2\end{array}\right)$.

$$
=5\left(\begin{array}{c}
n-3 \\
3
\end{array}\right) \text {. }
$$

By the similar operation, we get:

For $n-2 \geq r \geq 2, \quad N(3) C(n, \quad r)=r\left(\begin{array}{c}n-4 \\ r-2\end{array}\right)$.

For $n-3 \geq r \geq 2, \quad N(4) C(n, \quad r)=r\left(\begin{array}{c}n-5 \\ r-2\end{array}\right)$.

... $\ldots$

In this way

$$
N(t) C(n, r)=r\left(\begin{array}{c}
n-t-1 \\
r-2
\end{array}\right), n \geq r \geq 2, n-r+1 \geq t \geq 1
$$

2.2 Number of Occurrences of $t$ in All Compositions of $n$

Let the number be denoted by $N(t) C(n)$.

When $n \geq 1$ and $t=n$, then $N(t) C(n)=N(n) C(n)=1$.

The restrictions in (4) are:

$$
n \geq r \geq 2, \quad n-r+1 \geq t \geq 1 \quad \Leftrightarrow \quad n>t \geq 1, \quad n-t+1 \geq r \geq 2
$$

Hence for $n>t \geq 1$,

2.3 Other Results from (2), (4) and (5)

$$
\begin{aligned}
N(t) C(n) & =\sum_{r=2}^{n-t+1} N(t) C(n, r) \\
& =\sum_{r=2}^{n-t+1} r\left(\begin{array}{c}
n-t-1 \\
r-2
\end{array}\right) \\
\Rightarrow N(t) C(n) & =(n-t+3) 2^{n-t-2}, n>t \geq 1
\end{aligned}
$$

\section{(a) Number of summands in the compositions of $n$}

From (5), we get:

Number of the summands in the compositions of $n$ for $n \geq 2$

$=$ Number of occurrence of $t$ for $t=n+$ Number of occurrences of $t$ for $n>t \geq 1$

$$
\begin{gathered}
=1+\sum_{t=1}^{n-1}(n-t+3) 2^{n-t-2} \\
=1+2^{n-2}\left[(n+3) \sum_{t=1}^{n-1} 2^{-t}-\sum_{t=1}^{n-1} t 2^{-t}\right] \\
=(n+1) 2^{n-2}
\end{gathered}
$$

Obviously (6) holds for $n=1$ also.

(b) A proposition from (5)

Proposition 1. If $t_{1}$ and $t_{2}$ are the summands in the compositions of $n_{1}$ and $n_{2}$ respectively such that $n_{1}-t_{1}=n_{2}-t_{2}$, then the number of occurrences of $t_{1}$ in the compositions of $n_{1}$ is equal to the number of occurrences of $t_{2}$ in the compositions of $n_{2}$. 


\section{(c) Number-number relationship}

By Pascal's Identity, we get:

$$
r\left(\begin{array}{c}
n-2 \\
r-2
\end{array}\right)=r\left(\begin{array}{c}
n-1 \\
r-1
\end{array}\right)-r\left(\begin{array}{c}
n-2 \\
r-1
\end{array}\right)
$$

The above relation implies the following number-number relationship from (4) and (2).

Number of occurrences of 1 in $C(n, r)$

$=$ Number of the summands in $C(n, r)-$ Number of the summands in $C(n-1, r)$

\section{(d) Number-sum relationship}

From (6), we get a number-sum relationship as shown.

Number of the summands in the compositions of all $n$ integers: $1,2, \ldots, n$

$$
=\sum_{i=1}^{n}(i+1) 2^{i-2}
$$

$=$ Sum of the summands in the compositions of $n$.

\section{Counting for Complete Homogeneous Symmetric Polynomial: $h_{r}\left(x_{1}, \ldots, x_{k}\right)$}

\subsection{Number of Terms of the Polynomial}

The result is known. Here we count the number applying (2) and Vandermonde's identity. Let some terms of the polynomial contain some fixed $m$ of $k$ variables. The number of these terms $=N C(r, m)=\left(\begin{array}{c}r-1 \\ m-1\end{array}\right)$.

We have $k \gtreqless r$ in the problem. Hence we find: (i) either $1 \leq m \leq k<r$ (ii) or $1 \leq m \leq r \leq k$.

Case 1: When $1 \leq m \leq k<r$ then the number of terms

$$
\begin{gathered}
=\sum_{m=1}^{k}\left(\begin{array}{c}
k \\
m
\end{array}\right)\left(\begin{array}{c}
r-1 \\
m-1
\end{array}\right) \\
=\sum_{m=1}^{k}\left(\begin{array}{c}
k \\
k-m
\end{array}\right)\left(\begin{array}{c}
r-1 \\
m-1
\end{array}\right) \\
=\left(\begin{array}{c}
k+r-1 \\
k-1
\end{array}\right)=\left(\begin{array}{c}
k+r-1 \\
r
\end{array}\right)
\end{gathered}
$$

Case 2: When $1 \leq m \leq r \leq k$ then the number of terms

$$
\begin{gathered}
=\sum_{m=1}^{r}\left(\begin{array}{c}
k \\
m
\end{array}\right)\left(\begin{array}{c}
r-1 \\
m-1
\end{array}\right) \\
=\sum_{m=1}^{r}\left(\begin{array}{c}
k \\
m
\end{array}\right)\left(\begin{array}{c}
r-1 \\
r-m
\end{array}\right) \\
=\sum_{m=0}^{r}\left(\begin{array}{c}
k \\
m
\end{array}\right)\left(\begin{array}{c}
r-1 \\
r-m
\end{array}\right) \\
=\left(\begin{array}{c}
k+r-1 \\
r
\end{array}\right)
\end{gathered}
$$

It follows that the number of terms does not depend on equality or any inequality between $k$ and $r$, which are all taken into consideration in the process of solution. Thus we find:

\subsection{Number of Occurrences of an Integer $t$ as the Powers}

$$
\text { The number of terms of } h_{r}\left(x_{1}, \ldots, x_{k}\right)=\left(\begin{array}{c}
k+r-1 \\
r
\end{array}\right)
$$

Appling (4), we can count the number of occurrences of an integer $t$ as the powers in different terms among all $\left(\begin{array}{c}k+r-1 \\ r\end{array}\right)$ terms of $h_{r}\left(x_{1}, \ldots, x_{k}\right)$.

The condition of $t$ is: $r \geq t \geq 1$.

Case 1. The terms in which the integer $r$ occurs as the powers on the variables are: $x_{1}^{r}, \ldots, x_{k}^{r}$.

Therefore when $t=r$ then the number of occurrences of $t$ is $k$.

Case 2. When $t<r$, clearly then $r, k \neq 1$. From (4), we get:

The number of occurrences of $t$

$$
=\sum_{i=2}^{r} i\left(\begin{array}{l}
k \\
i
\end{array}\right)\left(\begin{array}{c}
r-t-1 \\
i-2
\end{array}\right), r, k \geq 2, \quad r-1 \geq t \geq 1
$$


(10) has some technical terms for some particular values of $k, r$ and $t$ such that the values of these terms are all 0 . The particulars in the context are described below.

(i) If $m$ is an integer in $(2, \ldots, r)$ then the product $m\left(\begin{array}{c}k \\ m\end{array}\right)\left(\begin{array}{c}r-t-1 \\ m-2\end{array}\right)$ or $m\left(\begin{array}{c}k \\ m\end{array}\right)\left(\begin{array}{c}r-t-1 \\ r-t+1-m\end{array}\right)$ is one among $r-$ 1 terms of (10). The value of the term is obviously 0 if $m>r-t+1$. For example, if the triplet $(k, r, t)$ is $(12,7$, $4)$ then the values of the last three terms of $(10)$ where $m \in(5,6,7)$ are all 0 . This implies that if the number of bases in a term of $h_{7}\left(x_{1}, \ldots, x_{12}\right)$ is 5,6 or 7 then the number of occurrences of 4 as the powers on the bases is 0 , or in other words 4 cannot occur as the powers on any of these bases.

(ii) When $r>k$ then the last $r-k$ terms have the factors: $\left(\begin{array}{c}k \\ k+1\end{array}\right), \ldots,\left(\begin{array}{l}k \\ r\end{array}\right)$ in succession such that the values of these $r-k$ terms are all 0 . In other words, for $r>k$, the number of occurrences of $t$ is equal to the summation: $\sum_{i=2}^{k} i\left(\begin{array}{c}k \\ i\end{array}\right)\left(\begin{array}{c}r-t-1 \\ i-2\end{array}\right)$.

\subsection{Number of Occurrences of a Variable $x_{m}$ as the Bases}

From Case 1 and Case 2 of Topic 3.2, we get:

Total number of bases in all terms of the polynomial

$$
=k+\sum_{t=1}^{r-1} \sum_{i=2}^{r} i\left(\begin{array}{l}
k \\
i
\end{array}\right)\left(\begin{array}{c}
r-t-1 \\
i-2
\end{array}\right)
$$

The number of occurrences of every variable $x_{m} \in\left(x_{1}, \ldots, x_{k}\right)$ in complete homogeneous symmetric polynomial of degree $r$ in the variables: $x_{1}, \ldots, x_{k}$ is same. Hence from (11.1), we get:

The number of occurrences of a variable $x_{m}$ as the bases

$$
=1+\frac{1}{k} \sum_{t=1}^{r-1} \sum_{i=2}^{r} i\left(\begin{array}{l}
k \\
i
\end{array}\right)\left(\begin{array}{c}
r-t-1 \\
i-2
\end{array}\right)
$$

\section{References}

Andrews, G. E. (2004). The Theory of Partitions, Cambridge University Press, Chapter 4, 54.

Arthur, T. B., \& Jennifer, Q. (2003). Proofs That Really Count: The Art of Combinatorial Proof, MAA, 63-68.

Hall, H. S., \& Knight, S. R. (1967). Higher Algebra, Macmillan and Co, London, 164 -166.

John, R. (1979). Combinatorial identities, R. E. Krieger Pub. Co.

Miklós, B. (2006). A walk through combinatorics, World Scientific Publishing Co. Pte. Ltd. 65-69, 89.

\section{Copyrights}

Copyright for this article is retained by the author(s), with first publication rights granted to the journal.

This is an open-access article distributed under the terms and conditions of the Creative Commons Attribution license (http://creativecommons.org/licenses/by/3.0/). 